highways and the byways. This defect, if defect it be, increases the responsibilities of the instructor, and urges the necessity of differentiation between fundamentals and accessories, as well as between a mere description of an experiment and its interpretation.

Viewed in its entirety the volume must unhesitatingly be pronounced to be a highly successful achievement of a highly difficult task. It is doubtless a much simpler as well as more agreeable undertaking to prepare a text-book in psychology than it is to sift out from endless sources and combine into a substantial compilation the facts upon which so much of psychological interpretation rests. It is particularly difficult to undertake this without guides or precedent; the successful achievement is the more notable by reason of the inherent difficulties. The present writer has used the manual in various stages of incompleteness as it appeared in the American Journal of Psychology and in advance sheets, for about five years in practical class-work and demonstrations, and is thus able to add to his appreciation of the scholarship of the compilation his verdict of its practical utility.

JOSERH JASTROW.

Handbuch der physiologischen Optik. H. von Helmholtz. Zweite umgearbeitete Auflage. Hamburg und Leipzig: Leopold Voss. I 896.

This is a very difficult book to review. Whenever one takes it up it is impossible not to be struck by some fascinating page which one has either not read before or not fully felt the meaning of, and that is an end of the critic's state of mind. But, in fact, there is no need of putting oneself into the critical frame of mind in noticing the new edition of the Physiologische Optik. With the completed volume before one, nothing but admiration can be felt for this model of the scientific method and the scientific spirit which has set a standard not easy to be reached again by the master of any science. Professor James has given expression to the common feeling in regard to this great work when he speaks of it as " a book which, on the whole, I imagine to be one of the four or five greatest monuments of human genius in the scientific line." The term genius is well chosen, for genius may be taken as connoting not simply great intellectual powers but a fusion of the quality of greatness in every element of the human being to whom the epithet is applied. It has lately been said by an acute observer that there is a common quality in the work of Darwin and that of Helmholtz, and a quality which is not only of the intellect, but of the spirit as well. Both writers have not only a complete mastery 
of their subject in all the ins and outs of its ramifications, but they have also that tone of mind which one comes nearest to describing if one calls it repose-that mental atmosphere which we recognize as natural in one who has gained mastery of execution in any form of art, but which we are not so prone to think possible in the intellectual workshop.

The first edition of this great work was issued between the years 1856 and 1867 ; the second has also been exactly eleven years in coming out $(1885-1896)$. For the first edition, Helmholtz undertook to work over, either by experiments of his own or by repeating the experiments of others, all the important points discussed; for the second edition a correspondingly enormous amount of labor could not be undertaken, but nevertheless it was designed that every important memoir that had appeared in the interval should be carefully considered, so far as it seemed to be an advance upon, or a desirable confirmation (or, as it might be, a refutation) of the results and opinions originally maintained. This plan was adhered to for the three parts first issued, but then the author's interest in the subject became aroused with something like its original intensity, and he began again to work at the solution of particular problems, without, indeed, experimenting himself, but basing his conclusions upon the experiments and measurements of others. In this way several memoirs were issued which were then, combined in part with the work of others, transferred almost literally to the out-coming volume. A notable paper of this category is the one on the connection between the external world and the data of the sense-organs. When the first edition of this work came out the doctrine of the empiricists had not been fully worked out; in the preface to that edition Helmholtz points out that other writers on this subject had treated only particular aspects of the question and that their views had not unnaturally been full of conspicuous contradictions; being himself of the opinion that, in a region in which disorders and disconnections prevail, even an untenable fundamental principle may have a useful function to perform in the marshaling and coördinating of facts, he gave a full exposition of the empirical theory, and in the course of this train of thought he became more and more convinced that it was the only one which could serve as an adequate guide through the wilderness of already known facts. In the present edition he holds to the same view with equal conviction, and his exposition is an admirable presentation of the best that can be done for that view. Nevertheless, in spite of the fact that the observer of each new case of a totally blind person who has 
been restored to sight gravely states that his case is a fresh confirmation of the empiristic theory, and in spite of the fact that the nativistic theory as expounded by Hering offers nothing that is to be preferred to it, it is certain that v. Helmboltz's view has quite lost its hold upon sound-thinking psychologists, and especially since the acute discussion of the subject which has been carried on in more recent times by Stumpf and James and Külpe.

It must also be admitted that the theory of the light-sense (as distinguished from the space-sense), which $v$. Helmholtz developed and defended, is considered at the present time by most of the psychologists to have very little in its favor. It is true that this is to be accounted for in part by the fact that the argument against that theory is patent to any one who is in the habit of rigorously analyzing sensation, while the argument for it is largely of a rather abstruse mathematical nature, and is not easily accessible to one who does not approach it with mathematical training. (So far as I can make out Dr. Scripture is the only pure psychologist who has given evidence of understanding the real force of this argument; nevertheless, it involves considerations which, as Dr. Scripture points out, are not elements of a theory merely, but plain-and most importantmatters of fact.) In this case also the victory seems not to be likely to go to the other of the two great contestants. Hering's theory, especially so far as it is a theory of assimilation and dissimilation, has always had to hold its own against a strongly antagonistic inertia on the part of the physiologists, and it shows no signs of overcoming that difficulty. Although the theory of Wundt has not proved particularly attractive to the attackers of theories, it is probable that by actual count it would be able to exhibit a greater number of adherents at the present time among those who are actively interested in the subject of theories (and these are, of course, the only ones whom it is worth while to take account of) than any of the others.

It is probable, therefore, that the two great doctrines which are most intimately associated with the name of Helmholtz, in the region of physiological optics, are destined to be given up in the near future, if they cannot be regarded as having been given up already. But what of that? It is the common fate of doctrines to be superseded by others better fitted to meet the needs of the time, and in this case the change of sentiment in these two particulars does very little to invalidate the claim of this book to be the indispensable and constant companion of whoever would concern himself with the subject of which it treats. It is a vast store-house of far-reaching, minute and 
thoroughly tested knowledge, and it will be very long before there is anything to take its place. In fact, one is sometimes brought to think that it is much too comprehensive; at least one poor writer for a German scientific journal has complained that he cannot get his contributions attended to because every one puts him off with the words: 'Es steht schon Alles im Helmholtz.' This comes very near, in fact, to being a literally true description of this monumental work.

It is to be regretted that Professor v. Helmholtz was not able to give one more year to the revision of this book; his death occurred in 1894 , and for a number of months before that time his attention had been so much absorbed in other branches of science-notably in meteorology - that he had practically abandoned doing any further work on the Optics. The last third of the book was brought out under the supervision of Professor König-of course, without changes from the original text. Professor König has also added a voluminous and carefully classified bibliography, covering some three hundred pages, and extending to the end of 1894 ; many of the investigations which have been incorporated into the body of the book are his work and that of his pupils. There is an editorial note of Professor König's on page roo8 which must not be overlooked, as it is of much importance. $\mathrm{He}$ points out that an error of calculation was afterwards detected in Helmholtz's determination of the fundamental colors by means of an extension of the psycho-physical law; that the master had regarded the investigation from the beginning rather as of the nature of a proof of the utility of the idea than as a means of arriving at definite results; and that he had come more and more to the conviction that the fundamental colors derived from their investigations by König and Dieterici not only met the conditions laid down by them, but were also consistent with a rigid upholding of the principle referred to.

It is also to be regretted that the revised volume had advanced so far that it was impossible to take account in it of the remarkable investigation by which Professor König has shown the coincidence between the relative absorption of light of different colors by the purple pigment of the rods and the relative luminosity of the spectrum as seen by the totally color-blind. v. Helmholtz had taken naturally the greatest interest in this investigation, and it would have constituted an important chapter of his book; this, together with the discovery by Hering of the identity of the vision of the totally color-blind with the night-vision of the normal eye may be regarded, doubtless, as the most important contributions made to psychological optics for many years. 
If Hering will probably fare no better than v. Helmholtz in regard to the two main points on which they have been at variance, there is a matter of minor interest concerning which his treatment is very much to be preferred to that of $v$. Helmholtz; in giving a mathematical discussion of the horopter, Hering has had the happy idea of making use of the methods of modern projective geometry. These were evidently wonderfully well adapted to the question, and they enable him to avoid the extremely long and complicated investigation of the question which is given by $\mathrm{v}$. Helmholtz.

\section{Ladd Franklin.}

\section{PSYCHICAL RESEARCH.}

\section{A Further Record of Observations of Certain Phenomena of} Trance. Richard Hodgson. Proceedings of Society for Psychical Research, Part XXXIII., Feb. 1898, Vol. XIII., pp. 284$5^{83}$.

A continuation of the case of the test-medium, Mrs. Piper, already reported on in previous 'Proceedings.' The present account is based on the results of 500 more sittings, about 130 of which were with unnamed strangers introduced to Mrs. Piper, for the first time. The almost exclusive 'control ' up to 1892 was a personality named Phinuit, concerning whose earthly identity no evidence has turned up. Since 1892 , however, the principal control has, until a year ago, purported to be the spirit of G. P., a young literary man recently dead in New York. The most striking feature of the present report is the expressed opinion of Dr. Hodgson, that the communications of G. P., as well as of others, now seem to him more naturally explicable on the hypothesis of spirit-return than on any other hypothesis. This conversion to spiritism of so critical an investigator, until lately disinclined to any such conclusion, marks, of course, the passage of a 'critical point' in the history of the Society for Psychical Research, as well as in Dr. Hodgson's own career.

The phenomenon, briefly described, is as follows: The medium waits passively for the trance to come on, which it now does quietly, though formerly there was a good deal of respiratory disturbance and muscular twitching. 'Phinuit' used to communicate entirely by speech, but G. P. early manifested himself by seeking to write on a pad placed on the medium's head. He now writes on the table. 'Phinuit' may talk whilst the hand is writing on other subjects, often 